\title{
REPRESENTATION AND CALCULATION METHOD OF PLANT ROOT
}

\author{
Hua Gao ${ }^{1, *}$, Yaqin Wang ${ }^{1,2}$, Zhijun Wang ${ }^{1}$ \\ ${ }^{1}$ College of Information Science \& Engineering, Shandong Agricultural University, Taian, \\ China, 271018 \\ ${ }^{2}$ College of Geo Info. Science and Technology, Shandong University of Science and \\ Technology, Tsingdao, China, 266510 \\ * Corresponding author, Address: College of Information Science \& Engineering, Shandong \\ Agricultural University, Taian, 271018, P. R. China, Tel: +86-538-8242497, Fax: + 86-538- \\ 8249275, Email: gaoh@sdau.edu.com
}

Abstract: In order to quantitatively analyze plant root growth and construct plant root growth model, a root system representation model based on tree-form data structure is proposed in this paper. After processing and analyzing root image, the information of root, such as length, diameter, number of child root, position of child root, angle of child root etc., is stored in a node of this treeform data structure. The accurate representation of root system is realized, which provides a foundation for the extraction and analysis of various root parameters. The specific algorithm is presented too.

Keywords: root image, representation model, tree-form data structure, image processing

\section{INTRODUCTION}

The architecture of plant root is the spatial configuration and distribution during its growth. It has an important effect on the nutrition and water absorption for plant from environment and soil. Studying the characteristics of plant root architecture can help people to deeply recognize plant root distribution, configuration, function, and to estimate the ability as well as to describe the process of plant root acquiring nutrition from soil in detail.

Root configuration includes stereo and planar configuration. Stereo configuration is a 3-D spatial distribution of different types of roots in 
medium. Planar geometric configuration is a 2-D planar distribution of various roots of the same root system along the root axis (Ge, 2002). In order to understand actual situation of growth and distribution of plant root in the soil, many measuring methods for root system have been studied (Luo, 2004). This kind of research has begun since 18 century.

The research of computer recognition for plant root growth characteristic has been paid a significant attention all over the world. Each country has made a different degree of progress (Zoom FC, 1990; Shuman LM, 1993; $\mathrm{Hu} \mathrm{XJ}, 2003)$. But the algorithm is not mature and the effect is not good.

In order to quantitatively analyze plant root growth and construct plant root growth model, an image processing-based plant root tree-form representation method is proposed in this paper. By using this method, the whole plant root architecture is expressed with a tree-form data structure. In this data structure, a root is represented with a node, and a child root is expressed with a child node. Experimental results show that this method can represent plant root architecture simply, naturally and accurately.

With above tree-form data structure, the root data can be stored into this structure. Based on image processing, the analysis and calculation of plant root can be implemented. Furthermore, an image thinning-based root system search algorithm is proposed in this paper. On the basis of binarization, filtering and thinning of original image, this method searches the whole root image with a breadth first search. After corresponding calculation and judgment, the calculation result information is stored into tree-form data structure. As a result, the representation of plant root is performed, which provides a solid foundation for the retrieval and analysis of various plant root parameters.

\section{PLANT ROOT TREE-FORM REPRESENTATION DATA STRUCTURE}

A multi-way tree structure is used to express a whole plant root. Here, main node expresses main root, while every child node expresses each child root. Main node and child node are represented with the same data structure, which includes description information and two chained lists. One chained list records detail information of current root, while the other records child root information.

Data structure of "expression tree" node:

$<$ child root ID>;

$<$ start point coordinates $>$;

$<$ length $>$;

$<$ included angle with parent root $>$;

$<$ expression chained list>;

$<$ number of child root>;

$<$ chained list of child root $>$. 
Data structure of expression chained list:

$<$ coordinates $>$;

$<$ width $>$.

Data structure of child root chained list:

$<$ child root $I D>$;

$<$ start point coordinates $>$.

With above data structure of expression tree, root information can be stored into this structure. In this paper, the measurement and calculation of plant root is realized based on image processing.

\section{ACQUISITION OF ROOT IMAGE}

It is the first step or even the very critical step for image processing and analysis to acquire appropriate and high quality image. The obtained root image should satisfy following conditions:

(1) It includes all the external figure information of measured root;

(2) It should be separated easily from background image;

(3) Useless background information should be reduced as much as possible.

Based on above three requests, it is needed to take following four measures when root image shooting.

(1) In order to separate root image from background easily, blue A4 paper is used as background, which has a bigger color difference with root system.

(2) In order to ensure measured root system has an even light requirement along shooting direction, so as to catch high quality image, incandescence lamp with diffusion property is used as light source.

(3) The measured root system should be laid as a plane surface when shooting. It is easy to realize. Generally speaking, common plant root is flexible. When the root is laid on the desk, it is nearly planar. Moreover, it is imperative that the root should be laid without crossover.

(4) The end of main root should be laid on the topside so as to be searched easily.

\section{IMAGE PREPROCESSING}

Firstly, the original image is processed by binarization, filtering and thinning to obtain a search image with only one single pixel width. See figure 1 to figure 3 . 

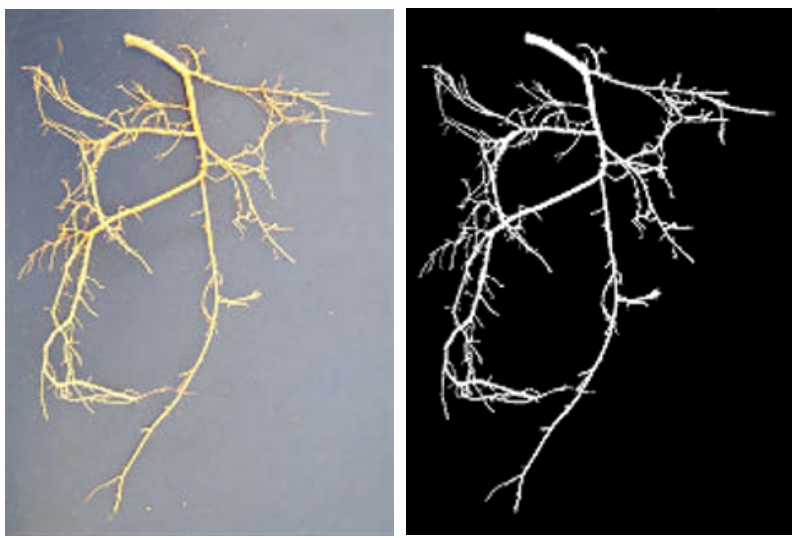

Figure 1. Original root image Figure 2. Binary root image

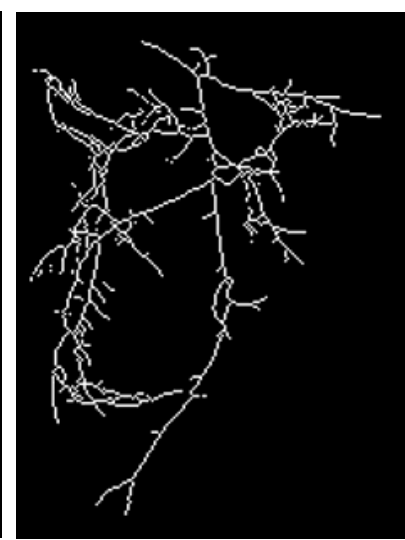

Figure 3. Thinning root image

\section{IMAGE SEARCH ALGORITHM}

The first step of this algorithm is finding start point of main root: When thinning image is scanned, the first point encountered is the start point of main root. Then, the root node of expression tree can be generated. The data is initialized as follows:

$<$ start point coordinate $>=$ start point coordinate;

$<$ length $>=0$;

$<$ included angle with parent root $>=0$;

$<$ expression chained list $>=$ empty;

$<$ child root number $>=0$;

$<$ child root chained list $>=$ empty

The search is begun. The algorithm searches the unlabeled point all the way. When a point is found, it will be labeled and be judged whether it has branches. See figure 4. In figure 4, $\square$ represents labeled pixel point, represents unlabeled pixel point, and $\boldsymbol{\Delta}$ represents current pixel point to be processed. In order to determine branch point, the number of 8neighborhood pixel point except labeled point of current pixel point is used. The method is: if current pixel point has no 8-neighborhood pixel point except labeled point, this point is end point. If there is one 8-neigborhood point, it has no branch. If there are two or more than two 8-neighborhood points, it is a branch point. There are different processing methods according to different number of branches. 


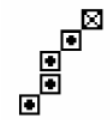

a

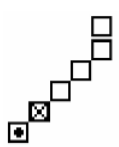

b

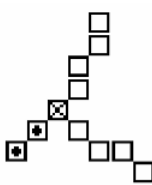

$\mathrm{c}$

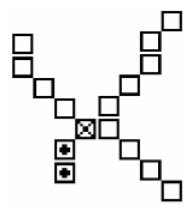

d

Figure 4. Sketch map of branch situation

(1) If current point is end point:

Here, current pixel point has no 8-neighborhood pixel point except labeled point. See figure 4(a).

Let's label this point;

$<$ length $>=<$ length $>+1$;

Add a node in $<$ expression chained list $>$;

$<$ coordinate $\rangle=$ current point coordinate;

$<$ width $>=1$;

After this node is processed, the brother node or child node will be processed. If all the nodes in this layer have been processed, the next one to be processed is child node. Otherwise, the next one is brother node.

(2) There is only one 8-neighborhood point:

It is obvious that this point has no branch. See figure 4(b).

Let's calculate the root diameter of this point. That is to say to calculate the root image width of this point. According to the coordinate of this point, the two boundary points on the binary image are searched. Then, the distance $\mathrm{D}$ between these two boundary points represents diameter of root in this point.

Let's label this point;

$<$ length $>=<$ length $>+1$;

Add a node in <expression chained list $>$;

$<$ coordinate $\rangle=$ current point coordinate;

$<$ width $>=\mathrm{D}$

The neighborhood point is used as current point.

(3) If there are two 8-neighborhood points:

It is obvious that there are two branches in this point. Then this point has a child root. See figure 4 (c).

At this time, it is needed to search the third point behind neighborhood point along two neighborhood points separately (if there are less than three points after neighborhood point, it seemed as noise and should be removed). Then the included angle between each branch and main root are calculated. Supposed that current point is A, the third point before current point is B,

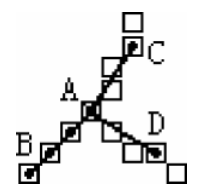

Figure 5. Main root and child root distinguishing in two branches 
and two third points of two branches are C and D separately. See figure 5 . The two included angles of two branches are as follows:

$$
\begin{aligned}
& \theta_{1}=\angle B A C=\cos ^{-1} \frac{A B^{2}+A C^{2}-B C^{2}}{2 A B \cdot A C} \\
& \theta_{2}=\angle B A D=\cos ^{-1} \frac{A B^{2}+A D^{2}-B D^{2}}{2 A B \cdot A D}
\end{aligned}
$$

Here $\mathrm{AB}, \mathrm{AC}, \mathrm{BC}$, and $\mathrm{BD}$ represent distance between two points.

Let's compare $\theta_{1}$ with $\theta_{2}$, the bigger one is main root, and smaller one is child root.

Let's label this point;

$<$ length $>=<$ length $>+1$;

Add a node in $<$ expression chained list $>$;

$<$ coordinate $>=$ current point coordinate;

$<$ width $>=\mathrm{D}$;

$<$ child root number $>=<$ child root number $>+1$

Add a node in $<$ child root chained list $>$;

$<$ child root number $>=$ previous $<$ child root number $>+1$;

$<$ start point coordinate $>=$ current point coordinate;

The neighborhood point with smaller included angle is used as current point.

(4) If there are three 8-neighborhood points, it shows that this point has three branches. See figure 4(d).

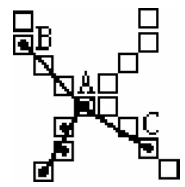

Figure 6. Three branches distinguishing

Now there are two situations. The first situation is that this point is the cross point of two roots. The middle path is current root. The other two paths form anther root. The second situation is that there are two child roots except main root. This can be judged according to the included angle of two paths. See figure 6.

$$
\theta=\angle B A C=\cos ^{-1} \frac{A B^{2}+A C^{2}-B C^{2}}{2 A B \cdot A C}
$$

If $\theta>165^{\circ}$, this point is a cross point of two roots. The other root need not be processed. The procedure is the same as step $\square$. But the tag of this point 
should be removed. If $\theta \leq 165^{\circ}$, this point has two child roots except main root. The node data should be modified (add two child roots).

To repeat above procedure until end point is found. After main root is processed, the child root of main root will be searched one by one. Because there may exist loop during the search, the breadth first search method instead of deep first search is used.

\section{CONCLUSIONS}

The root system is fully represented by tree-form data structure. The information such as root length, root diameter, number of child root, position of child root, angle of child root etc. is stored in a node. The accurate representation of root system is realized, which provides a foundation for the extraction and analysis of various root parameters.

However, the research of root system is on a plane surface in this paper. Further research can be finished on the stereo image of root system to realize analysis of stereo spatial configuration of root system.

Moreover, the algorithm proposed in this paper can only detect root configuration parameters of tap root system. It needs to be perfected in future, so as to realize the measurement of root configuration parameter for every kind of root system.

\section{REFERENCES}

Ge Zhenyang, Yan Xiaolong, Luo Xiwen 2002, Simulation Models of Plant Root System Architecture and Application: A Review. Trans. CSAE. 18 (3):154-160.

Hu Xiujuan 2003, The Application of Digit Image Treatment Technique in Plant Root Study. Forestry Machinery \& Woodworking Equipment. 31 (3):29-31.

Luo Xiwen, Zhou Xuecheng, Yan Xiaolong 2004, Visualization of Plant Root Morphology in situ Based on X-ray CT Imaging Technology. Transactions of the Chinese Society for Agricultural Machinery. 35 (2):104-106.

Shuman L.M, Ramseur E.L, Wilson, D 1993, Video image method compared to a hand method for determining root lengths. Journal of Plant Nutrition. 16 (4):563-571.

Zoom F.C, van Tienderen P.H 1990, A rapid quantitative measurement of root length and root branching by microcomputer image analysis. Plant and Soil. 126:301-308. 